\title{
Xanthogranulomatous Cystitis Presenting as Urinary Incontinence and a Vesicovaginal Fistula: A Case Report and Review of the Literature
}

\author{
Shivon N. Abdullah, Adamantios M. Mellis, Corinne L. Puzio, Daniel J. Culkin* \\ Department of Urology, University of Oklahoma Health Sciences Center, Oklahoma City, USA \\ E-mail:*Daniel-Culkin@ouhsc.edu
}

Received June 7, 2011; revised July 15, 2011; accepted July 28, 2011

\begin{abstract}
Anthogranulomatous cystitis (XC) is an inflammatory condition of the urinary bladder that is benign and rarely seen. XC is a unique disease in which there have been only 26 cases described in the literature, including our case. Patients with XC often present with lower urinary tract symptoms, hematuria, abdominal pain, or abdominal mass. We present the unusual case of an 81 year old female who presents with urinary incontinence, which was later diagnosed as a vesicovaginal fistula. After describing the treatment of this patient, a review of the literature is detailed including presentation, possible etiologies, and treatment of XC.
\end{abstract}

Keywords: Xanthogranulomatous Cystitis, Inflammation, Bladder, Vesicovaginal Fistula, Urinary Incontinence

\section{Introduction}

Xanthogranulomatous cystitis is an uncommon but benign inflammatory condition of the bladder that is chronic in duration for which the etiology is unknown [1]. XC was first described by Ota in 1933[2]. Histologically, the disease is characterized by multinucleated giant cells and chronic inflammation. The characteristic cell is the lipid-laden macrophages, known as "foamy histiocytes," which stain strongly for CD 68. Although the kidney is the most common site of xanthogranulomatous disease, there are currently twenty-five previously described cases of this disease reported in the bladder [3]. Most of these cases involve urachal remnants with disease most often isolated to the dome of the bladder [4]. We add our case of XC in an 81 year old female with the unusual initial presentation of urinary incontinence and vesicovaginal fistula (VVF) and provide a brief review of the literature

\section{Case Report}

An 81 year old Caucasian female of German descent was referred to clinic with primary complaint of continuous urinary incontinence which had persisted for several months and believed to be secondary to stress urinary incontinence. The patient also had symptoms of urinary frequency and intermittent gross hematuria. However, the patient denied having any urinary tract infections. She also reported a significant unintentional weight loss in the past year. Past medical history included hypertension, diabetes mellitus type II, as well as dyslipidemia.

On physical examination, the patient was found to have a large tumor involving the anterior vaginal wall with pooling of urine in the vaginal vault, suspicious for a vesicovaginal fistula. Laboratory analysis revealed normal renal function, absence of hematologic abnormalities, including eosinophilia. Urinalysis and urine culture failed to show to urinary tract infection. Contrast computed tomography with urogram phase revealed a midline posterior bladder wall lesion and a $4 \mathrm{~mm}$ communication between the posterior bladder wall and the anterior vaginal wall (Figures 1 and 2).

Cystoscopy was performed identifying significant amount of bullous edema as well as a bulky solid lobulated mass approximately $2 \mathrm{~cm}$ in size in the area of the left posterior bladder neck extending to the trigone of the bladder. A midline vesicovaginal fistula was identified in the posterior bladder wall. The lateral walls of the bladder, as well as the dome of the bladder were free of masses or inflammation. Due to concern for malignancy, endoscopic biopsy and resection were performed of the 


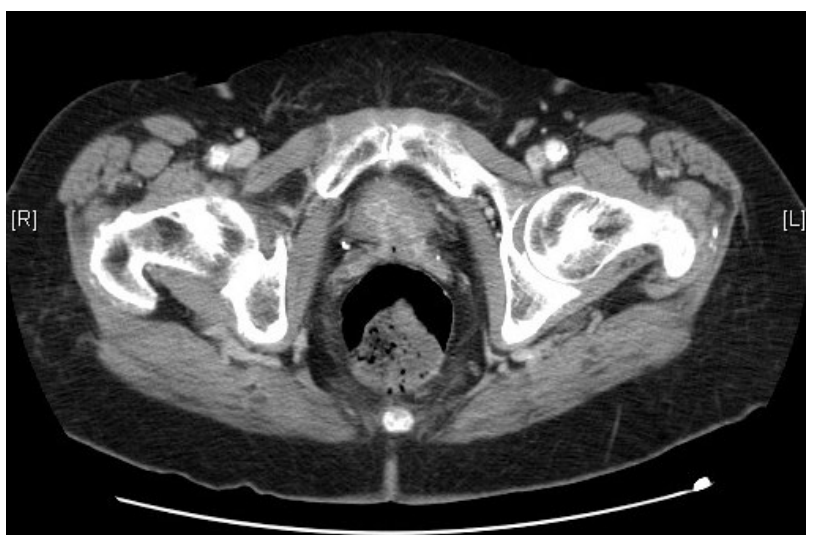

Figure 1. Contrast CT of the pelvis demonstrates a discernable mass in the area of the bladder trigone extending into the anterior vaginal wall.

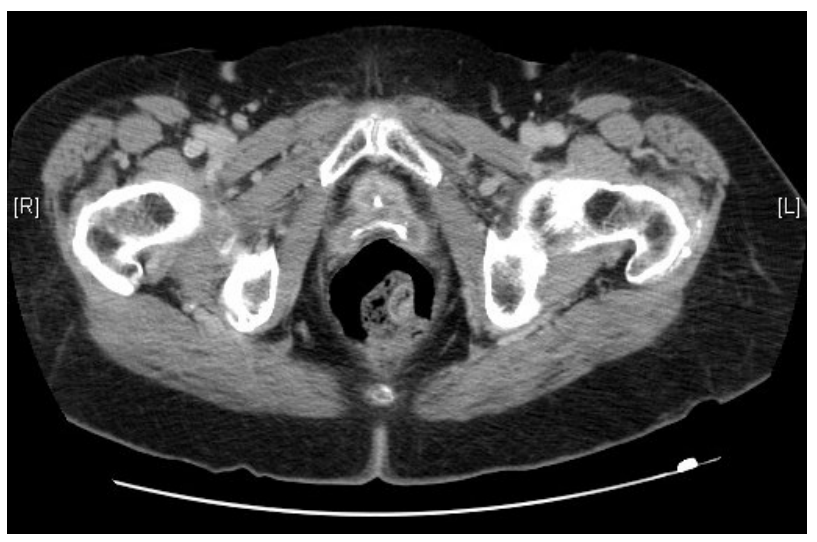

Figure 2. Delayed phase contrast CT of the pelvis demonstrates vesicovaginal fistula with presence of contrast within the vaginal vault.

bladder neck lesion. Vaginoscopy was also performed with biopsies of the anterior vaginal wall and fistulous tract also obtained. Histologic analysis revealed numerous foamy histiocytes. Immunohistochemical staining demonstrated positive staining for CD68 (Figure 3) a known marker for macrophages.

The patient was treated conservatively with oral antibiotics for five months with resulting decrease in inflammation; however, urinary incontinence persisted. As a result, the patient elected to undergo vesicovaginal fistula repair. Intraoperatively, attempted vaginal vesicovaginal fistula repair was halted due to extensive fibrosis at the bladder neck and trigone of the bladder. Then an abdominal approach to VVF repair was approached. However, the right ureteral orifice was unable to be cannulated, and, on further exploration, the patient was found to have right hydroureteronephrosis. Furthermore, extensive fibrosis was present in the pelvis, the bladder neck and trigone, making vaginal and suprapubic VVF repair not possible.

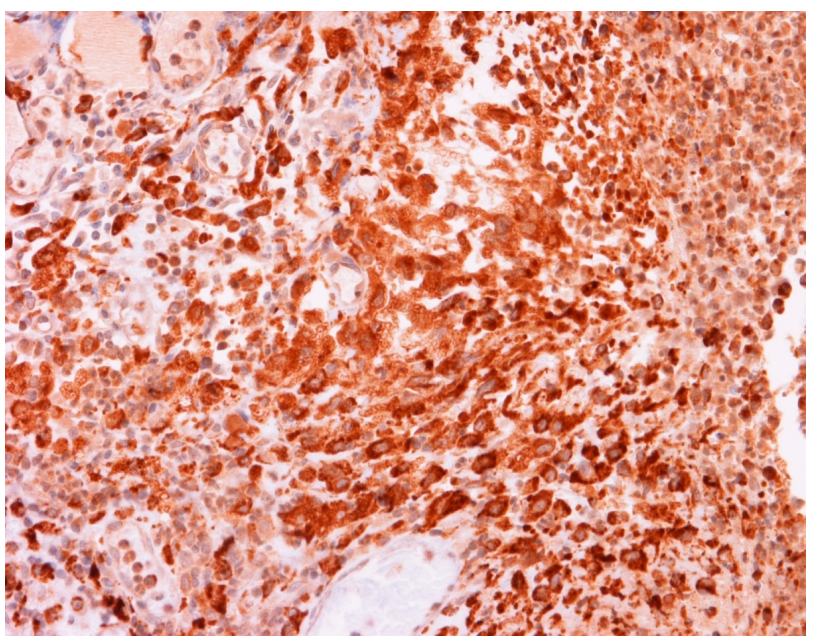

Figure 3. CD68 immunohistochemical staining demonstrating numerous characteristic "foamy histiocytes" and multinucleated giant cells in the submucosa of the bladder (CD68 immunostain $\times 400$ ).

With those surgical findings and taking the patient's primary complaint of incontinence into consideration, the decision was made that the patient would best benefit from a urinary diversion procedure. An ileal conduit urinary diversion was performed. The patient was discharged after five days in the hospital, and now, more than 3 years later, she is asymptomatic and is pleased with her continence.

\section{Discussion}

Xanthogranulomatous cystitis is a rare disease entity and only 26 cases have been reported and described, includeing this case. XC's etiology is unknown. Some authors have proposed chronic irritation and inflammation of a urachal remnant [2], autoimmune etiology as demonstrated by eosinophilia [5], as well as chronic urinary tract infections and anaerobic infections [6]. Regardless of the true etiology, the ages of patients afflicted with the disease range from 16-80 years of age, and include both males and females in equal distribution [4]. Patients typically present with lower urinary tract symptoms $60 \%$ $(15 / 25)$ of the time and symptoms include frequency, urgency, as well as nocturia. Hematuria (microscopic or gross) is present in about a quarter of the patients (6/25), whereas abdominal or pelvic pain is present in $40 \%$ $(12 / 25)$ of patients. Moreover, an abdominal or pelvic mass is identified in $40 \%(12 / 25)$ of patients [3-9]. Table 1 provides a summary of these statistics.

When the location of the disease was provided in the case reports, the majority of cases involved the bladder dome and was associated with an urachal remnant or anomaly: $80 \%(16 / 20)$ and 56\% (14/25), respectively. 
Table 1. Symptoms seen in patients affected by xanthogranulomatous cystitis [4,5].

\begin{tabular}{cc}
\hline Symptoms & Percentage (\%) \\
\hline LUTS & $60(15 / 25)$ \\
Hematuria (gross and microscopic) & $24(6 / 25)$ \\
Pain (abdominal and pelvic) & $40(12 / 25)$ \\
Mass (abdominal and pelvic) & $40(12 / 25)$ \\
Urachal Remnant Involved & $56(14 / 25)$ \\
\hline
\end{tabular}

Other areas reported to be involved include the posterior wall (1 case), lateral walls ( 2 cases), and the anterior wall ( 2 cases).

The treatment modality most often employed in cases of XC is partial cystectomy, as is the case in 18 out of 25 cases reported (72\%). Other XC cases have been successfully managed with surgical or endoscopic resection and recurrences have never been reported, even 15 years after initial resection [1]. Other treatment modalities that have also been curative are long-course antibiotics and radical cystectomy [8-9].

Our case is rather unique in that our patient is the oldest patient (81 years old) reported to be diagnosed with $\mathrm{XC}$. Furthermore, the patient possesses a unique presentation of XC. This is the first case of XC that has presented as urinary incontinence, which was diagnosed as a vesicovaginal fistula. Furthermore, our patient is the first patient where the disease has involved the bladder trigone and the bladder neck. Management of this patient is also distinctive, given her unusual case. The patient's $\mathrm{XC}$ was primarily treated with long term antibiotics, while her secondary problem was treated with urinary diversion with both modalities resulting in successful treatment of a complicated disease process. As in other cases, our patient has remained asymptomatic. Although resection was not undertaken, chronic antibiotic treatment seemed to have decreased the inflammation caused by the disease, supporting the case for possible bacterial infection as a contributing etiology [6].

In conclusion, xanthogranulomatous cystitis is a rare disease that manifests in a variety of ways, including in our case, as chronic bladder inflammation leading to development of a vesicovaginal fistula.

\section{References}

[1] L. B. Tan, C. P. Chiang, C. H. Huang and C.-H. Chian, "Xanthogranulomatous Cystitis: A Case Report and Review of the Literature," International Urology Nephrology and Nephrology, Vol. 26, No. 4, 1994, pp. 413-417. doi:10.1007/BF02768011

[2] N. Ota, "Urachal Tumor of Inflammatory Origin,” Grangigebie, Vol. 7, 1933, p. 1205.

[3] D. E. Chung, L. K. Carr, L. Sugar, M. Hladunewich and L. A. Deane, "Xanthogranulomatous Cystitis Associated with Inflammatory Bowel Disease,” Canadian Urological Association Journal, Vol. 4, No. 4, 2010, pp. E91E93.

[4] A. Fornari, M. Dambros, C. Teloken, A. A. Hartmann, J. Kolling and R. Seben, "A Case of Xanthogranulomatous Cystitis,” International Urogynecology Journal, Vol. 18, No. 10, 2007, pp. 1233-1235. doi:10.1007/s00192-007-0345-5

[5] M. K. Chung, M. Y. Seol, W. Y. Cho, H. K. Seo and J. S. Kim, "Xanthogranulomatous Cystitis Associated with Suture Material,” The Journal of Urology, Vol. 159, No. 3, 1998, pp. 981-982. doi:10.1016/S0022-5347(01)63794-7

[6] H. L. Tai, C. C. Chen and K. T. Yeh, "Xanthogranulomatous Cystitis Associated with Anaerobic Bacterial Infection,” The Journal of Urology, Vol. 162, No. 3 Pt 1, 1999, pp. 795-796.

[7] S. K. Singh, A. K. Khandelwal, D. S. Pawar R. Sen and S. Sharma, "Xanthogranulomatous Cystitis: A Rare Clinical Entity,” Urology Annals, Vol. 2, No. 3, 2010. pp. 125126. doi:10.4103/0974-7796.68863

[8] R. Goel, G. Kadam, A. Devra, S. Patel and P. Modi, "Xanthogranulomatous Cystitis,” International Urology Nephrology and Nephrology, Vol. 39, No. 2, 2007, pp. 477478. doi:10.1007/s11255-006-9059-8

[9] S. Ekici, E. Dogan, S. Ruacan and A. Midi, "Xanthogranulomatous Cystitis: A Challenging Imitator of Bladder Cancer,” The Scientific World Journal, Vol. 10, 2010, pp. 1160-1173. doi:10.1100/tsw.2010.139

\author{
Abbreviations: \\ $\mathrm{XC}$ - xanthogranulomatous cystitis; \\ VVF-vesicovaginal fistula; \\ CT_-computer tomography.
}

